\title{
Una aplicación de lógica difusa en el modelado de una cadena de suministro con incertidumbre en el abastecimiento de materiales
}

\author{
María Laura Cúnico y Aldo Vecchietti \\ INGAR (CONICET-UTN), Avellaneda 3657, Santa Fe (3000), Argentina
}

\begin{abstract}
El problema que se aborda en este artículo tiene como objetivo la optimización de la compra de materiales y entrega de productos en la cadena de suministros, considerando fallas en la provisión de materias primas por parte de los proveedores. El modelo matemático que se formula es mixto entero nolineal (MINLP) y abarca un horizonte de tiempo dividido en varios períodos. Para fortalecer la relación con los proveedores se les ofrece contratos de compra, por material adquirido, en cada uno de los períodos propuestos. Para el modelado se recurre a la utilización de conjuntos difusos (fuzzy sets) que permiten asignar grados de confiabilidad a cada proveedor a partir del comportamiento histórico registrado por los mismos. El modelo determina los proveedores y las cantidades comprar y cuáles son los contratos a ofrecer de modo tal de minimizar los costos de compra y almacenamiento de materias primas. El modelo se emplea para realizar un análisis de sensibilidad del proceso de compra y la relación con sus proveedores. Para ello se realiza se proponen varios escenarios fijando la periodicidad de falla de cada proveedor, con estos valores fijos, el modelo MINLP se transforma en uno lineal MILP (Mixed Integer Linear Program) en cada uno de los escenario propuesto. El objetivo principal del artículo radica entonces en exponer una perspectiva distinta a la existente en la literatura cuando la provisión incierta de materiales no presenta una distribución de falla conocida o cuando un enfoque probabilístico no refleja eficientemente el comportamiento de cada proveedor. La resolución de los escenarios propuestos permite visualizar la dependencia del proceso de compra con los proveedores de materias primas.
\end{abstract}

Keywords: Fuzzy Sets, programación matemática difusa, demanda incierta, selección de proveedores y contratos.

\section{Introducción}

El deseo de insertarse en el mercado actual exige incrementar la competitividad, promoviendo la necesidad de fortalecer los vínculos existentes entre los distintos agentes que interactúan en una cadena de suministros. El propósito de una cadena de suministros es mejorar la eficiencia global de la red que la componen los proveedores, la empresa y sus clientes. La coordinación entre las diferentes unidades que componen la cadena de suministros se puede alcanzar por medio de un intercambio de

adfa, p. 1, 2011.

(C) Springer-Verlag Berlin Heidelberg 2011 
información apropiado o estableciendo compromisos, por medio de la forma de contratos, entre la empresa y sus proveedores ó la empresa y sus clientes [1].

Una situación común que se presenta en las empresas de producción es la falta de provisión de materias primas, que afectan la producción de bienes y las ventas, traduciéndose en demandas insatisfechas y la insatisfacción de los clientes. La falta de abastecimiento puede deberse a diversos factores, proveedores que han sufrido algún accidente de importancia en sus plantas, otros que dejaron de producir algunos bienes, algunos materiales en los que la demanda supera a la oferta.

Existen diversos trabajos en la literatura abierta que tratan la firma de contrato con los proveedores para disminuir la incertidumbre en la entrega. Park y otros [2] estudiaron el proceso de compra por medio de un modelo de programación disyuntiva, enfocándose en el proceso de selección de proveedores y de contratos de compra a los mismos. Ellos mostraron que la firma de contratos es una práctica de negocio que contribuye a disminuir la incertidumbre en la provisión de materias primas. Naraharisetti y otros [3], así como Laínez y otros [4] establecen que la firma de contratos con los proveedores es una decisión estratégica que puede ser fundamental para disminuir los riesgos en la cadena de suministros. Rodriguez y Vecchietti [5] presentaron una formulación para la selección de contratos de compra en los casos que existen incertidumbres en la provisión. La incertidumbre se maneja asumiendo una distribución de falla conocida para cada uno de los proveedores. Como resultado de la ejecución de este problema se determina los proveedores con quienes firmar los contratos, el tipo de contrato a firmar y las cantidades involucradas en los mismos.

Este artículo muestra una perspectiva distinta a la presentada por Rodriguez y Vecchietti [5] para los casos en que no es factible establecer una distribución de falla en el abastecimiento de los proveedores. Impulsado por la evidente influencia que ejerce la demanda insatisfecha de materiales sobre la calidad de servicio ofrecido, este trabajo propone un modelo de programación matemática lineal basada en conjuntos difusos (fuzzy sets). Se hace un análisis de sensibilidad, asumiendo que cada proveedor tiene una periodicidad de falla perteneciente a diferentes sectores del conjunto difuso que define un porcentaje de error en su entrega. A partir de este análisis se determina la dependencia de la empresa con los proveedores y sus fallas. El objetivo es determinar la selección óptima de proveedores para minimizar el impacto ocasionado por incumplimientos en la entrega de las órdenes de compra solicitadas. En la vida real se presentan situaciones que no pueden ser representadas eficientemente a partir de enfoques determinísticos ó probabilísticos, en este caso el empleo de los conjuntos difusos pueden brindar información beneficiosa para la gestión de la cadena de suministros.

El artículo presenta la siguiente estructura: en la sección 2 se introduce y describe brevemente el problema a tratar. A continuación, en la sección 3 se desarrolla el modelo, exhibiendo las explicaciones que motivan la definición de cada restricción. En la sección 4, se despliega un caso de estudio y sus resultados, el cual permite ilustrar el funcionamiento del modelo propuesto en el apartado anterior. Por último, las conclusiones son presentadas en la sección 5 . 


\section{Descripción del problema}

El problema que se aborda en este artículo tiene como objetivo la optimización de la compra de materiales y entrega de productos en la cadena de suministros, en un horizonte de tiempo dividido en varios períodos. El problema también considera la selección del contrato de compra por material adquirido en cada período propuesto.

Los materiales con características semejantes son agrupados en familias, debiendo satisfacer la demanda por período de cada una de ellas. Cada proveedor exhibe una capacidad máxima de entrega por material y se suponen conocidos los precios correspondientes en cada caso. Estos importes se mantienen inalterables en los distintos períodos de tiempo considerados.

En general, se supone que todo proveedor tiene la capacidad de suministrar cualquier material en cada período de tiempo.

Cada proveedor presenta además una periodicidad de falla en la entrega parcial o total de los materiales pedidos. Dichas fallas son modeladas a partir de funciones difusas que dependen del riesgo de incumplimiento de cada proveedor.

El modelo decide el material y el proveedor que deberá abastecerlo junto con la cantidad requerida y el tipo de contrato que se firma para realizar la transacción. Cada uno de los tres tipos de contratos considerados $\left(c_{1}, c_{2}, c_{3}\right)$, representa una política de compra distinta cuya flexibilidad influye en el cálculo de los costos finales. En todos los casos, se prevé una cantidad mínima de compra que depende del proveedor seleccionado y del tipo de contrato que define la relación comercial. Más aún, en el primer caso se otorga el menor descuento ofrecido debido a que el número de unidades exigidas para la compra también es mínimo. En el segundo caso, se incrementa la obligación de compra así como también la tasa de descuento sobre el costo de los materiales ordenados. Este comportamiento se atribuye a la intención de generar una relación comercial a largo plazo entre el comprador y el proveedor, que se percibe al limitar la elección de este tipo de contrato al caso de que el material solicitado haya sido provisto por el mismo proveedor en el período anterior. Por último, el tercer tipo de contrato incluye la mayor exigencia de compra y un aumento sobre el importe total, debido a la flexibilidad admitida en el pago. Se establece que la cancelación de la deuda generada por la compra de materiales se realice dos períodos posteriores a la entrega de los mismos.

\section{Formulación del Problema}

La representación del modelo incluye variables binarias de decisión tanto para la elección de proveedores como de los respectivos contratos a establecer. Notar también que, de la característica de pago diferido que se establece en el contrato $c_{3}$ se desprende la necesidad de definir un número superior de intervalos de tiempo, digamos $t_{m}$, donde $m>n$ y $n$ es el número total de períodos considerados sobre el horizonte de planificación fijado.

Satisfacción de Demanda y Stock

La ecuación 1 determina que la cantidad pedida de todos los materiales de la familia $f$ para todo proveedor $j$ en el período $t, q(j, k, t)$, más la cantidad en stock de 
dicha familia en el mismo período $t, s(f, t)$, debe ser mayor o igual a la demanda de la familia $f$ en el período considerado, $F D(f, t)$.

$\sum_{k \in F K}(f, k) \sum_{j} q(j, k, t)+s(f, t) \geq F D(f, t) \quad, \forall f \epsilon F, \forall t \leq t_{n+1}$

Para brindar cierta flexibilidad al plan de compra se define una cota superior, $U B>0$, que representa el exceso de material permitido. Por tanto, la cantidad de materiales pedidos pertenecientes a $f$ más los existentes en stock en el período $t$ no deben superar la demanda de dicha familia más el excedente preestablecido:

$\sum_{k \epsilon F K(f, k)} \sum_{j} q(j, k, t)+s(f, t) \leq F D(f, t) *(1+U B) \quad, \forall f \epsilon F, \forall t \leq t_{n+1}$

El stock a principio del período $t$ de cada familia $f$ se calcula como la suma del stock a principio del período inmediatamente anterior y la cantidad esperada de todos los proveedores, eqq( $f, t-1)$, menos las ventas de la familia $f$ en el mismo período $t-1, d(f, t-1)$, como se observa en la ecuación 3 .

$s(f, t)=s(f, t-1)+e q q(f, t-1)-d(f, t-1), \forall f \epsilon F, \forall t \geq t_{1} \vee \forall t \leq t_{m}$

Dado que la capacidad de almacenaje es limitada, la restricción 4 exige que el stock de materiales de todas las familias en cada período de tiempo $t$ sea inferior a la capacidad máxima de stock conocida $S C$.

$\sum_{f \in F} S(f, t) \leq S C \quad, \forall t \leq t_{n+1}$

Por otro lado, en el período $t_{1}$ el stock de la familia $f$ está determinado por el stock inicial $I S(f)$ registrado en dicho período y que se introduce como un parámetro del problema.

$s\left(f, t_{1}\right)=I S(f), \forall f \epsilon F$

Por último en la ecuación 6 se determina que las ventas de la familia $f$ en el período $t$ deben ser menores o iguales que la demanda que presenta esta familia en el intervalo de tiempo mencionado.

$d(f, t) \leq F D(f, t) \quad, \forall f \epsilon F, \forall t \in T$

\section{Selección de Proveedores y Pedidos}

En las ecuaciones 7-12 se introducen variables binarias de decisión, $y_{1}(j, f, t)$ e $y_{2}(j, f, k, t)$, que valen 1 si el proveedor $j$ es seleccionado para satisfacer los pedidos por familia y por material, respectivamente.

Las ecuaciones 7 y 8 establecen que es posible comprar al proveedor $j$ los materiales $k$ pertenecientes a la familia $f$ en el período $t, y_{2}(j, f, k, t)$, sólo si $j$ ha sido seleccionado para abastecer a esta familia, $y_{1}(j, f, t)$, en algún período del horizonte de tiempo. Notar que el lado izquierdo de la ecuación 7 puede ser mayor que 1 si el proveedor $j$ ha sido seleccionado para abastecer a la familia $f$, debido a que puede proveer más de un material que pertenece a esta familia. Por lo tanto, para lograr esta flexibilidad se emplea el producto de la variable binaria $y_{1}(j, f, t)$ y el mayor número de materiales pertenecientes a todas las familias de $F$. 
$\sum_{k \in F K(f, k)} y_{2}(j, f, k, t) \leq \max _{f \in F}|f| * y_{1}(j, f, t) \quad, \forall j \epsilon J, \forall f \epsilon F, \forall t \leq t_{n+1}$

$y_{2}(j, f, k, t)=0 \quad, \forall j \in J, \forall f \notin F K(f, k) \vee t \geq t_{n+1}$

Mediante la ecuación 9 , se determina que el pedido del material $k$ se realizará al proveedor $j$ si este ha sido elegido para suministrar a la familia $f$ a la que pertenece $k$. Por lo tanto $q(j, k, t)$ será mayor que cero sólo si $y_{1}(j, f, t)$ es igual a 1 y estará acotada superiormente por el número máximo de unidades de $k$ que pueden ser abastecidas por $j$.

$q(j, k, t) \leq Q \max (j, k) * y_{1}(j, f, t), \forall j \in J, \forall k \in F K(f, k), \forall f \in F, \forall t \leq t_{n+1}$

Además, la cantidad de materiales de la familia $f$ ordenados en el período $t$ al proveedor $j$, debe ser menor o igual a la capacidad máxima de entrega que $j$ admite para la respectiva familia.

$\sum_{k \in F K(f, k)} q(j, k, t) \leq \sum_{k \in F K(f, k)} \operatorname{Qmax}(j, k) * y_{1}(j, f, t)$

$\forall j \epsilon J, \forall f \epsilon F, \forall t \leq t_{n+1}$

Más aún, se exige que la cantidad ordenada de material $k$ no supere la capacidad máxima registrada por el proveedor $j$ al que se le realiza el pedido.

$q(j, k, t) \leq Q \max (j, k) * y_{2}(j, f, k, t), \forall j \epsilon J, \forall k \in F K(f, k), \forall f \epsilon F, \forall t \leq t_{n+1}$

Asignación de Contratos

Para determinar el tipo de contrato seleccionado para realizar la transacción de compra se introducen variables binarias de decisión $y_{3}\left(j, c, k, t_{1}\right)$ que valen 1 si el contrato $c$ es elegido para comprar el material $k$ al proveedor $j$ en el período $t$.

Si el proveedor $j$ ha sido elegido en el período $t$ para proveer el material $k$ de la familia $f$, entonces debe seleccionarse un único contrato, $y_{3}(j, \mathrm{c}, k, t)$, para realizar la transacción de compra, como se establece en la ecuación 12.

$\sum_{c \epsilon C} y_{3}(j, c, k, t)=y_{2}(j, f, k, t) \quad, \forall j \epsilon J, \forall k \in F K(f, k), \forall f \in F, \forall t \leq t_{n+1}$

Las ecuaciones 13 y 14 se incorporan debido a que, el tipo de contrato $c_{2}$ sólo puede ser seleccionado cuando el material $k$ ha sido adquirido del mismo proveedor en el período previo $t-1$.

$y_{3}\left(j, c_{2}, k, t_{1}\right)=0 \quad, \forall j \epsilon J, \forall k \epsilon K$

$y_{3}\left(j, c_{2}, k, t\right) \leq \sum_{c \epsilon C} y_{3}(j, c, k, t-1) \quad, \forall j \epsilon J, \forall k \in K, \forall t \leq t_{n+1}$

Además, los pedidos están limitados por un número mínimo de unidades, el cual depende del contrato y el proveedor seleccionado para la compra. Por tanto, la ecuación 15 establece que la cantidad ordenada de material $k$ debe ser mayor o igual a la cantidad mínima que se precisa en el contrato $c$ si la compra se realizará a $j$. Notar que, si el contrato $c$ no es seleccionado, la ecuación 15 resulta redundante como consecuencia de la utilización de una expresión Big-M. 
$q(j, k, t) \geq Q \min (c, j)-B M\left(1-y_{3}(j, c, k, t)\right), \forall j \epsilon J, \forall k \epsilon K, \forall c \epsilon C, \forall t \epsilon T$

Modelado de Fallas

Como se ha mencionado en la sección anterior, la falla en la cantidad de material entregado por cada proveedor $j, \mu_{j}(\cdot)$, se modela a partir de una función de pertenencia que depende del riesgo o periodicidad de incumplimiento de $j, p(j)$.

En este caso, la clasificación del desempeño de cada proveedor se divide en tres categorías que coinciden con los intervalos de incertidumbre fijados para este caso $\left(I_{1}^{j}, I_{2}^{j}, I_{3}^{j}\right)$ : "Regular" si $j$ falla en la entrega de la totalidad del material ordenado $\left(p(j) \epsilon I_{1}^{j}\right)$, "Excelente" si $j$ entrega por completo el pedido recibido $\left(p(j) \epsilon I_{3}^{j}\right)$ y "Bueno" si $j$ realiza una entrega parcial de los materiales solicitados $\left(p(j) \epsilon I_{2}^{j}\right)$.

Por lo tanto resulta que, la proporción de unidades que no son entregadas por $j$ se representa por una función continua y definida a trozos de la siguiente manera:

$$
\mu_{j}(p(j))= \begin{cases}1 & , \text { si } p(j) \epsilon I_{1}^{j} \\ f_{j}(p(j)) & , \text { si } p(j) \epsilon I_{2}^{j} \\ 0 & , \text { si } p(j) \epsilon I_{3}^{j}\end{cases}
$$

donde $f_{j}(\cdot)$ es una función lineal decreciente, y los intervalos de incertidumbre $I_{r}^{j}, 1 \leq r \leq 3$ se describen como $I_{1}^{j}=\left(0, p_{j}^{L}\right), I_{2}^{j}=\left[p_{j}^{L}, p_{j}^{U}\right]$ y $I_{3}^{j}=\left(p_{j}^{U}, 1\right)$, siendo $p_{j}^{L}, p_{j}^{U}$ datos del problema que representan respectivamente las cotas inferior y superior de $p_{j}, \forall j \in J$. Notar que el número de intervalos definidos y sus respectivas funciones $f_{j}(\cdot)$, pueden variar dependiendo de la clasificación, el grado de detalle y la complejidad que sea posible de alcanzar, a partir del estudio del comportamiento histórico de cada proveedor.

A partir de la combinación de las categorizaciones definidas se especifican distintos escenarios, con el objetivo de analizar la influencia que ejerce cada proveedor sobre los costos de compra, y determinar de este modo las relaciones comerciales más relevantes. De aquí se desprende entonces que la periodicidad de falla de cada proveedor, $p(j), j \epsilon J$, se transforman en parámetros fijos en cada uno de los escenarios propuestos, resultando conocidos también los intervalos $I_{r}^{j}, 1 \leq r \leq 3$, a los que pertenecen. Por lo tanto, para cada $j \epsilon J, \mu_{j}(\cdot)$ es un parámetro que se calcula a partir de las ecuaciones 16 y 17.

$$
f_{j}(p(j))=\frac{1}{p_{j}^{L}-p_{j}^{U}} * p(j)-\left(\left(\frac{1}{p_{j}^{L}-p_{j}^{U}}\right) * p_{j}^{U}-1\right), \forall j \epsilon J
$$

Una vez modelada la falla, es posible determinar la cantidad de materiales de cada familia que se espera recibir del proveedor $j, e q q_{j}(f, t)$, como se observa en (18).

$$
e q q_{j}(f, t)=\sum_{k \epsilon F K(f, k)} p(j) * q(j, k, t) *\left(1-\mu_{j}\right) \quad, \forall f \epsilon F, \forall t \epsilon T, \forall j \epsilon J
$$


Observar que, como se ha remarcado anteriormente, el deseo de realizar un análisis de sensibilidad basado en escenarios de los efectos que producen los lazos comerciales entre proveedores-empresa, resulta que tanto $p(j)$ como $\mu_{j}$ son parámetros del modelo planteado y por lo tanto la ecuación 18 es lineal.

Así, la cantidad esperada de la familia $f$ en el período $t$ de todos los proveedores es igual a la suma de las cantidades esperadas de cada proveedor, como se muestra en la ecuación 19.

$e q q(f, t)=\sum_{j \epsilon J} e q q_{j}(f, t)$

$\underline{\text { Restricciones de Costo }}$

Las ecuaciones 20 y 21 determinan que el precio de compra del material $k$ ordenado al proveedor $j$ en el período $t$ bajo el contrato $c, w(j, k, c, t)$, es igual a la cantidad ordenada de $k$ por el precio regular de venta ofrecido por el proveedor $j$, menos el beneficio establecido en el contrato $c$ sobre el importe total. Esto se ha introducido mediante expresiones Big-M que transforman ambas ecuaciones en redundantes en caso de no haber seleccionado el contrato $c$ para realizar dicha orden.

$$
\begin{aligned}
& w(j, k, c, t) \leq q(j, k, t) * P C(j, k) *(1-\delta(j, c))+B M *\left(1-y_{3}(j, c, k, t)\right) \\
& w(j, k, c, t) \geq q(j, k, t) * P C(j, k) *(1-\delta(j, c))-B M *\left(1-y_{3}(j, c, k, t)\right) \\
& \quad \forall j \in J, \forall k \epsilon K, \forall c \epsilon C, \forall t \in T
\end{aligned}
$$

Se añade también una restricción que fuerza al precio de compra del material $k$ a ser nulo en el caso de que el proveedor $j$ no haya sido seleccionado para suministrar este material.

$w(j, k, c, t)=B M * y_{3}(j, c, k, t) \quad, \forall j \epsilon J, \forall k \epsilon K, \forall c \epsilon C, \forall t \epsilon T$

Dado que el tipo de contrato $c_{3}$ prevé una política de pago diferido, resulta que la suma a abonar en el período $t$ debida a la compra de material $k, m(j, c, k, t)$, es igual al precio de compra de $k$ en el período $t_{\theta}=t+\theta, \theta>0$. En otro caso, si el tipo de contrato seleccionado es $c_{1}$ o $c_{2}$ el monto a pagar coincide con el precio de compra del material $k$ en el mismo período, es decir, $t_{\theta}=t$.

$$
\begin{aligned}
& m\left(j, c_{3}, k, t_{\theta}\right)=w\left(j, c_{3}, k, t\right), \forall j \epsilon J, \forall k \epsilon K, \forall t \epsilon T, t_{\theta}>t \\
& m(j, c, k, t)=w(j, c, k, t), \forall j \epsilon J, \forall k \in K, \forall t \epsilon T, \forall c \epsilon C, c \neq c_{3}
\end{aligned}
$$

\section{$\underline{\text { Restricciones Adicionales }}$}

Las ecuaciones 25-27 son presentadas para calcular el stock promedio de la lia $f$ en el período $t, \operatorname{savg}(f, t)$, que se utilizará posteriormente en la función objetivo. Entonces,

$$
\operatorname{savg}(f, t)=\frac{\sum_{e \epsilon E} s d(f, e, t)}{e_{h}} \quad, \forall f \epsilon F, \forall t \epsilon T
$$


donde $s d(f, e, t)$ representa el stock de la familia $f$ en cada subperíodo $e$ del período $t$, calculado como la diferencia entre el stock de la familia $f$ en el subperíodo $e-1 \mathrm{y}$ un coeficiente de salida de stock dado por $s(f, t) / e_{h}$.

$s d(f, e, t)=s d(f, e-1, t)-\frac{s(f, t)}{e_{h}} \quad, \forall f \in F, \forall t \leq t_{n}, \forall e \in E=\left\{e_{2}, \cdots, e_{h}\right\}$

$s d\left(f, e_{1}, t\right)=s(f, t), \forall f \epsilon F, \forall t \in T$

Función Objetivo

La función objetivo, presentada en la ecuación 28, apunta a minimizar los costos debidos a las compras de materiales, el costo de inventario y el costo por la pérdida de ventas ocasionadas por la demanda de materiales insatisfecha.

$\sum_{t \in T} \frac{\sum_{j \epsilon J} \sum_{k \in K} \sum_{c \epsilon C} m(j, c, k, t)+\sum_{f \in F} A P(f, t) *(F D(f, t)-d(f, t))+\sum_{f \in F} M S * \operatorname{savg}(f, t) * \operatorname{costavg}(f)}{(1+R R)^{t}}$

donde $A P(f, t)$ es el precio de venta promedio de la familia $f$ en período $t, M S$ es un porcentaje del costo del material crudo, $\operatorname{costavg}(f)$ es el costo promedio de la familia $f$ y $R R$ es una tasa de retorno correspondiente al costo de capital.

El modelo matemático final está constituido por las ecuaciones (1)-(28).

\section{Caso de Estudio}

El caso de estudio propuesto consiste en 32 materiales $(K)$, agrupados en 10 familias $(F)$, a ser aprovisionadas por 4 potenciales proveedores $(J)$ con distintas proporciones de fallas en la entrega de las ordenes solicitadas. En todos los casos, se definen tres intervalos de incertidumbre mediante los cuales se clasifica la entrega de materiales como total $\left(I_{3}^{j}, 1 \leq j \leq 4\right)$, parcial $\left(I_{2}^{j}, 1 \leq j \leq 4\right)$ o nula $\left(I_{1}^{j}, 1 \leq j \leq 4\right)$. Los extremos de estos intervalos se presentan en la tabla 1 .

Table 1. Intervalo de incertidumbre del proveedor $j$

\begin{tabular}{c|cccc}
\hline \multirow{2}{*}{ Intervalos } & \multicolumn{4}{|c}{ Proveedores } \\
\cline { 2 - 5 } & $\boldsymbol{j}_{\mathbf{1}}$ & $\boldsymbol{j}_{\mathbf{2}}$ & $\boldsymbol{j}_{\mathbf{3}}$ & $\boldsymbol{j}_{\mathbf{4}}$ \\
$\boldsymbol{p}_{\boldsymbol{j}}^{\boldsymbol{L}}$ & 0.25 & 0.30 & 0.27 & 0.33 \\
$\boldsymbol{p}_{\boldsymbol{j}}^{\boldsymbol{U}}$ & 0.75 & 0.70 & 0.73 & 0.67 \\
\hline
\end{tabular}

Por otro lado, en la tabla 2 se exhibe la relación de los materiales con sus familias $F K(f, k)$, el stock inicial $I S(f)$ y el precio de venta promedio por familia por período de tiempo $A P(f, t)$, mientras que en la tabla 3 se presentan las exigencias contractuales de compra $Q \min (c, j)$ y las tasas de beneficio por contrato $\delta(j, c)$.

Se supone también que el horizonte de tiempo propuesto se divide en tres períodos $\left(t_{n}, n=3\right)$, cada uno particionado en 30 subperíodos $\left(e_{h}\right)$. 
Como se ha mencionado en las secciones previas, se consideran tres tipos de contratos $\left(c_{1}, c_{2}, c_{3}\right)$. Notar que de la última política de compra, que se desprende de $c_{3}$, surge la necesidad de definir el conjunto de períodos como:

$$
T=\left\{t_{1}, \cdots, t_{n}, \cdots, t_{m} \mid t_{m}=t_{n+2} \wedge n=3\right\}
$$

Table 2. Composición, stock inicial y precio de la familia $f, F K(f, k), I S(f)$ y $A P(f, t)$

\begin{tabular}{|c|c|c|c|c|c|}
\hline \multirow[t]{2}{*}{ Familias } & \multirow{2}{*}{$\begin{array}{c}\text { Materiales } \\
F K(f, k)\end{array}$} & \multirow{2}{*}{$\begin{array}{r}\text { Stock } \\
I S(f)\end{array}$} & \multicolumn{3}{|c|}{$\begin{array}{c}\text { Precio promedio de venta } \\
(A P(f, t))\end{array}$} \\
\hline & & & $t_{1}$ & $t_{2}$ & $t_{3}$ \\
\hline$f_{1}$ & $k_{1}, k_{2}, k_{3}$ & 40 & 0.90 & 0.90 & 0.90 \\
\hline$f_{2}$ & $k_{4}, k_{5}, k_{6}, k_{7}$ & 45 & 0.92 & 0.92 & 0.92 \\
\hline$f_{3}$ & $k_{8}, k_{9}, k_{10}$ & 40 & 1.10 & 1.10 & 1.10 \\
\hline$f_{4}$ & $k_{11}, k_{12}, k_{13}$ & 40 & 0.90 & 0.90 & 0.90 \\
\hline$f_{5}$ & $k_{14}, k_{15}, k_{16}, k_{17}$ & 40 & 0.93 & 0.93 & 0.93 \\
\hline$f_{6}$ & $k_{18}, k_{19}, k_{20}$ & 30 & 1.12 & 1.12 & 1.12 \\
\hline$f_{7}$ & $k_{21}, k_{22}$ & 32 & 0.74 & 0.74 & 0.74 \\
\hline$f_{8}$ & $k_{23}, k_{24}, k_{25}$ & 40 & 1.11 & 1.11 & 1.11 \\
\hline$f_{9}$ & $k_{26}, k_{27}, k_{28}, k_{29}$ & 30 & 0.95 & 0.95 & 0.95 \\
\hline$f_{10}$ & $k_{30}, k_{31}, k_{32}$ & 45 & 1.21 & 1.21 & 1.21 \\
\hline
\end{tabular}

Table 3. Cantidad mínima exigida de compra según contrato c al proveedor $j(\boldsymbol{Q} \min (\boldsymbol{c}, \boldsymbol{j}))$ y tasa de descuento o interés de contrato c $(\boldsymbol{\delta}(\boldsymbol{j}, \boldsymbol{c}))$

\begin{tabular}{c|cccc|cccc|}
\hline \multirow{3}{*}{$\boldsymbol{C}$} & \multicolumn{4}{|c|}{$\operatorname{Qmin}(c, j)$} & \multicolumn{4}{c|}{$\delta(j, c)$} \\
\cline { 2 - 9 } & \multicolumn{4}{c|}{ Proveedores } & \multicolumn{4}{c|}{ Proveedores } \\
\cline { 2 - 9 } $\boldsymbol{c}_{\mathbf{1}}$ & $\boldsymbol{j}_{\mathbf{1}}$ & $\boldsymbol{j}_{\mathbf{2}}$ & $\boldsymbol{j}_{\mathbf{3}}$ & $\boldsymbol{j}_{\mathbf{4}}$ & $\boldsymbol{j}_{\mathbf{1}}$ & $\boldsymbol{j}_{\mathbf{2}}$ & $\boldsymbol{j}_{\mathbf{3}}$ & $\boldsymbol{j}_{\mathbf{4}}$ \\
$\boldsymbol{c}_{\mathbf{2}}$ & 50 & 40 & 32 & 38 & 0.12 & 0.06 & 0.095 & 0.055 \\
$\boldsymbol{c}_{\mathbf{3}}$ & 70 & 60 & 52 & 55 & 0.15 & 0.12 & 0.15 & 0.121 \\
\hline
\end{tabular}

Además, se fijan como parámetros de entrada la capacidad de stock $(S C)$ en 5000 unidades, la cota superior de la demanda $(U B)$ en 0.5 , el porcentaje de costo de material crudo $(M S)$ como 0.25 y la razón de retorno $(R R)$ en 0.15 .

Por último, la tabla 4 muestra para cada proveedor su capacidad máxima de entrega y el precio de compra de cada material. 
Table 4. Cantidad máxima de material $k$ ordenada a j en el periodo $t(\boldsymbol{Q} \max (\boldsymbol{j}, \boldsymbol{k}))$ y precio regular del material $k$ vendido por el proveedor $j(\boldsymbol{P C}(\boldsymbol{j}, \boldsymbol{k}))$

\begin{tabular}{|c|c|c|c|c|c|c|c|c|}
\hline \multirow{3}{*}{$\boldsymbol{K}$} & \multicolumn{4}{|c|}{$\operatorname{Qmax}(j, k)$} & \multicolumn{4}{|c|}{$P C(j, k)$} \\
\hline & \multicolumn{4}{|c|}{ Proveedores } & \multicolumn{4}{|c|}{ Proveedores } \\
\hline & $\begin{array}{l}\boldsymbol{j}_{\mathbf{1}} \\
150\end{array}$ & $\begin{array}{c}\boldsymbol{j}_{2} \\
160\end{array}$ & $\begin{array}{c}\boldsymbol{j}_{3} \\
150\end{array}$ & $\begin{array}{c}\boldsymbol{j}_{4} \\
160\end{array}$ & $\begin{array}{l}\boldsymbol{j}_{1} \\
0.5\end{array}$ & $\begin{array}{l}\boldsymbol{j}_{2} \\
0.52\end{array}$ & $\begin{array}{l}\boldsymbol{j}_{\mathbf{3}} \\
0.51\end{array}$ & $\begin{array}{l}\boldsymbol{j}_{4} \\
0.55\end{array}$ \\
\hline$k_{2}$ & 50 & 70 & 55 & 50 & 0.47 & 0.49 & 0.48 & 0.45 \\
\hline$k_{3}$ & 50 & 65 & 45 & 64 & 0.8 & 0.81 & 0.79 & 0.82 \\
\hline $\boldsymbol{k}_{4}$ & 145 & 180 & 165 & 175 & 0.71 & 0.78 & 0.69 & 0.70 \\
\hline$k_{5}$ & 60 & 80 & 65 & 60 & 0.75 & 0.72 & 0.73 & 0.77 \\
\hline$k_{6}$ & 50 & 80 & 40 & 77 & 0.51 & 0.52 & 0.53 & 0.54 \\
\hline$k_{7}$ & 60 & 50 & 53 & 52 & 0.49 & 0.45 & 0.48 & 0.47 \\
\hline$k_{8}$ & 158 & 160 & 159 & 142 & 0.78 & 0.68 & 0.72 & 0.70 \\
\hline$k_{9}$ & 60 & 45 & 50 & 40 & 0.72 & 0.81 & 0.71 & 0.70 \\
\hline$k_{10}$ & 65 & 60 & 66 & 70 & 0.75 & 0.74 & 0.72 & 0.79 \\
\hline$k_{11}$ & 45 & 80 & 42 & 70 & 0.45 & 0.55 & 0.52 & 0.51 \\
\hline$k_{12}$ & 148 & 150 & 140 & 160 & 0.46 & 0.48 & 0.47 & 0.48 \\
\hline$k_{13}$ & 50 & 50 & 60 & 22 & 0.78 & 0.81 & 0.88 & 0.81 \\
\hline$k_{14}$ & 160 & 150 & 162 & 180 & 0.70 & 0.72 & 0.71 & 0.75 \\
\hline$k_{15}$ & 50 & 45 & 20 & 48 & 0.80 & 0.75 & 0.74 & 0.72 \\
\hline$k_{16}$ & 60 & 50 & 49 & 60 & 0.51 & 0.54 & 0.50 & 0.51 \\
\hline$k_{17}$ & 70 & 66 & 65 & 73 & 0.47 & 0.57 & 0.48 & 0.49 \\
\hline$k_{18}$ & 170 & 150 & 175 & 175 & 0.8 & 0.78 & 0.79 & 0.77 \\
\hline$k_{19}$ & 60 & 65 & 50 & 63 & 0.71 & 0.73 & 0.71 & 0.75 \\
\hline$k_{20}$ & 70 & 75 & 70 & 72 & 0.75 & 0.70 & 0.75 & 0.72 \\
\hline$k_{21}$ & 180 & 185 & 150 & 180 & 0.51 & 0.52 & 0.50 & 0.50 \\
\hline$k_{22}$ & 70 & 75 & 60 & 70 & 0.49 & 0.48 & 0.45 & 0.50 \\
\hline$k_{23}$ & 165 & 152 & 145 & 165 & 0.79 & 0.78 & 0.78 & 0.81 \\
\hline$k_{24}$ & 60 & 65 & 70 & 70 & 0.71 & 0.70 & 0.72 & 0.73 \\
\hline$k_{25}$ & 85 & 82 & 80 & 84 & 0.73 & 0.71 & 0.73 & 0.72 \\
\hline$k_{26}$ & 170 & 175 & 180 & 185 & 0.51 & 0.52 & 0.53 & 0.50 \\
\hline$k_{27}$ & 85 & 80 & 90 & 80 & 0.47 & 0.45 & 0.48 & 0.49 \\
\hline$k_{28}$ & 110 & 100 & 125 & 105 & 0.78 & 0.81 & 0.82 & 0.81 \\
\hline$k_{29}$ & 95 & 110 & 104 & 100 & 0.81 & 0.71 & 0.75 & 0.72 \\
\hline$k_{30}$ & 125 & 120 & 130 & 120 & 0.77 & 0.74 & 0.75 & 0.75 \\
\hline$k_{31}$ & 110 & 100 & 100 & 105 & 0.80 & 0.82 & 0.88 & 0.85 \\
\hline$k_{32}$ & 80 & 82 & 80 & 75 & 0.81 & 0.80 & 0.83 & 0.84 \\
\hline
\end{tabular}

Por último, se fijan como parámetros de entrada la capacidad de stock $(S C)$ en 5000 unidades, la cota superior de la demanda $(U B)$ en 0.5 , el porcentaje de costo de material crudo $(M S)$ como 0.25 y la razón de retorno $(R R)$ en 0.15 . 


\section{Solución del caso estudio}

Para realizar el análisis de sensibilidad se definieron 12 escenarios cuya configuración se expone en la tabla 5. A partir de estos datos se calculan los porcentajes de falla en la entrega de materiales $\mu_{j}$ que aparecen en la tabla 6.

El modelo presentado en las secciones previas fue planteado en GAMS (General Algebraic Modeling System) y ejecutado en una computadora personal con procesador Intel Core i7. El tiempo de resolución de los 12 escenarios, utilizando el resolvedor GUROBI, requirió aproximadamente de un minuto y se desprenden varias decisiones a partir de aquí. En primer lugar, se determina una selección óptima de proveedores para satisfacer los pedidos de materiales en todo el horizonte de tiempo. Luego se escoge el tipo de contrato a utilizar para la compra de cada material ordenado y las cantidades solicitadas.

Table 5. Periodicidad de falla del cada proveedor en los distintos escenarios

\begin{tabular}{c|cccc}
\hline \multirow{2}{*}{ Escenarios } & \multicolumn{4}{|c}{ Razón de falla } \\
\cline { 2 - 5 } E1 & $\boldsymbol{p}\left(\boldsymbol{j}_{\mathbf{1}}\right)$ & $\boldsymbol{p}\left(\boldsymbol{j}_{\mathbf{2}}\right)$ & $\boldsymbol{p}\left(\boldsymbol{j}_{\mathbf{3}}\right)$ & $\boldsymbol{p}\left(\boldsymbol{j}_{\mathbf{4}}\right)$ \\
E2 & 0.15 & 0.45 & 0.65 & 0.95 \\
E3 & 0.15 & 0.65 & 0.95 & 0.15 \\
E4 & 0.15 & 0.95 & 0.15 & 0.45 \\
E5 & 0.45 & 0.15 & 0.65 & 0.95 \\
E6 & 0.45 & 0.65 & 0.95 & 0.15 \\
E7 & 0.45 & 0.95 & 0.15 & 0.65 \\
E8 & 0.65 & 0.15 & 0.45 & 0.95 \\
E9 & 0.65 & 0.45 & 0.95 & 0.15 \\
E10 & 0.65 & 0.95 & 0.15 & 0.45 \\
E11 & 0.95 & 0.15 & 0.45 & 0.65 \\
E12 & 0.95 & 0.45 & 0.65 & 0.15 \\
\hline
\end{tabular}

Los costos totales registrados en cada escenario considerado, junto con los proveedores seleccionados y sus respectivos porcentajes de falla en la entrega de materiales $\left(\mu_{j}\right)$ se exhiben en la tabla 6.

Table 6. Resumen de resultados

\begin{tabular}{|l|l|l|l|l|l|l|}
\hline $\begin{array}{c}\text { Escena- } \\
\text { rios }\end{array}$ & $\begin{array}{c}\text { Función } \\
\text { Objetivo }\end{array}$ & $\boldsymbol{\mu}_{\mathbf{1}}$ & $\boldsymbol{\mu}_{\mathbf{2}}$ & $\boldsymbol{\mu}_{\mathbf{3}}$ & $\boldsymbol{\mu}_{\mathbf{4}}$ & $\begin{array}{c}\text { Provee- } \\
\text { dores }\end{array}$ \\
\hline $\mathrm{E} 1$ & 1666.91 & 1 & 0.62 & 0.17 & 0 & $\mathrm{j} 3, \mathrm{j} 4$ \\
$\mathrm{E} 2$ & 1682.37 & 1 & 0.12 & 0 & 0.64 & $\mathrm{j} 3$ \\
$\mathrm{E} 3$ & 1659.04 & 1 & 0 & 0.61 & 0.05 & $\mathrm{j} 2, \mathrm{j} 3, \mathrm{~J} 4$
\end{tabular}




\begin{tabular}{|l|l|l|l|l|l|l|} 
E4 & 1666.91 & 0.60 & 1 & 0.17 & 0 & $\mathrm{j} 3, \mathrm{j} 4$ \\
E5 & 1682.37 & 0.60 & 0.12 & 0 & 1 & $\mathrm{j} 3$ \\
E6 & 1657.87 & 0.60 & 0 & 1 & 0.05 & $\mathrm{j} 1, \mathrm{j} 2, \mathrm{~J} 4$ \\
E7 & 1666.13 & 0.20 & 1 & 0.61 & 0 & $\mathrm{j} 1, \mathrm{j} 4$ \\
E8 & 1682.37 & 0.20 & 0.62 & 0 & 1 & $\mathrm{j} 3$ \\
E9 & 1651.52 & 0.20 & 0 & 1 & 0.64 & $\mathrm{j} 1, \mathrm{j} 2$ \\
E10 & 1665.61 & 0 & 1 & 0.61 & 0.05 & $\mathrm{j} 1$ \\
E11 & 1665.61 & 0 & 0.62 & 0.17 & 1 & $\mathrm{j} 1$ \\
E12 & 1665.61 & 0 & 0.12 & 1 & 0.64 & $\mathrm{j} 1$ \\
\hline
\end{tabular}

Las celdas sombreadas indican, en la segunda columna, los costos máximo(E2, E5, E8) y mínimo(E9) hallados mediante la implementación del modelo propuesto. Del mismo modo, se han resaltado los porcentajes de falla de cada proveedor (de la tercer a la sexta columna) en aquellos escenarios en los que han sido seleccionados (por ejemplo, el proveedor 1 se escoge en los escenarios E6-E7 y E9-E12).

Se observa de los resultados expuestos que los menores costos registrados se alcanzan en los escenarios E9, E6 y E3 respectivamente, en los cuales la falla en la entrega de materiales del proveedor $j_{2}$ es nula. Más aún, el proveedor $j_{2}$ sólo es considerado como una opción viable cuando su desempeño es "Excelente".

Por otro lado, el máximo costo registrado se alcanza en aquellos escenarios en los cuales se ha seleccionado como único proveedor a $j_{3}$ ( E2, E5 y E8), el cual garantiza la entrega total de la orden en el $95 \%$ de los casos.

Esta marcada influencia que ejerce $j_{2}$, minimizando los costos totales al no incurrir en incumplimientos en la entrega de materiales, evidencia la necesidad de fortalecer los vínculos comerciales con este proveedor, con el propósito de disminuir futuras fluctuaciones en los costos.

\section{Conclusiones}

En este artículo se presenta una aplicación de la lógica difusa en el modelado de una cadena suministro con aprovisionamiento incierto. Debido a la estrecha relación existente entre la calidad de servicio prestada y las utilidades percibidas, resulta eminente apelar a prácticas que permitan determinar distintas estrategias para optimizar la coordinación entre los diversos agentes que conforman la cadena. En muchas ocasiones, no es factible establecer el déficit en el cumplimiento de algún agente de manera determinística o probabilística, pero se cuenta con una clasificación de este comportamiento que brinda información relevante para el funcionamiento de la empresa. Es por ello que se recurre a la incorporación de conjuntos borrosos, a partir de los cuales es posible lograr una representación apropiada en este tipo de casos.

Un problema que comúnmente se presenta en las empresas es el desabastecimiento de materias primas, el cual afecta tanto a la producción como a la comercialización, disminuyendo la eficiencia frente a otros competidores del mercado. Una herramienta utilizada para salvar esta dificultad es la firma de contratos de compras para minimi- 
zar la incertidumbre presente en la entrega de materiales. El presente trabajo abarca esta problemática apuntando a determinar, mediante un análisis de escenarios, el conjunto de proveedores que al incurrir en una falla, ejercen mayor influencia sobre los beneficios y fijar en cada caso un tipo de contrato apropiado para formalizar la transacción. De este modo se logran reconocer los proveedores más influyentes con los cuales resulta esencial promover el fortalecimiento de los lazos comerciales.

\section{Referencias}

1. Guillén, G.; Mele, F.; Espuña A.; Puigjaner, L. Addressing the Design of Chemiscal Supply Chains under Demand Uncertainty. Ind. Eng, Chem. Res. 2006, 45, 7566-7581.

2. Park, M.; Park, S.; Mele, F.; Grossmann, I. E. Modeling of purchase and sales contracts in supply chain optimization.Ind. Eng. Chem. Res. 2006, 45, 5013.

3. Naraharisetti, P. K.; Adhitya, A., Karimi, I.; Srinivasan, R. Decision support for resilient enterprisess-From suppliers to shareholders. Presented at Foundations of Computer-Aided Process Operations (FOCAPO 2008), Boston, MA, Jun 29-Jul 2, 2008.

4. Laínez, J. M; Puigjaner, L.; Reklaitis, G. V. Financial and financial engineering considerations in supply chain and product development pipeline management. Presented at Foundations of Computer-Aided Process Operations (FOCAPO 2008), Boston, MA, Jun 29-Jul 2, 2008.

5. Rodriguez, M.A; Vecchietti, A. Logical and Generalized Disjunctive Programming for Supplier and Contract Selection under Provision Uncertainty. Ind. Eng. Chem. Res. 2009, 48, 5506-5521.

6. Thakre, P.A. ; Shelar, D.S. ; Thekre S.P. Solving fuzzy linear programming problem as multiobjetive linear programming problem. Journal of Engineering and Technology Research, May 82-85, 2009. 